\title{
Editorial: Beyond Cognitive Ability
}

\author{
Srecko Joksimovic ${ }^{1}$, George Siemens ${ }^{2}$, Yuan Wang ${ }^{3}$, Maria Ofelia Z. San Pedro ${ }^{4}$, Jason Way ${ }^{5}$
}

${ }^{1}$ Address: Centre for Change and Complexity in Learning, University of South Australia, BH 321 Barbara Hanrahan Adelaide, South Australia ${ }^{2}$ Address: Centre for Change and Complexity in Learning, University of South Australia, BH 321 Barbara Hanrahan Adelaide, South Australia; School of Psychology, University of Texas Arlington, 323 Nedderman Hall, Arlington, Texas, 76019, USA

Corresponding Author: ${ }^{3}$ Email: elle.wang@asu.edu Address: Arizona State University, EdPlus, Suite 200, Tempe, Arizona, 85251, USA

${ }^{4}$ Address: ACT, Inc., 500 ACT Drive, lowa City, lowa, 52243, USA

${ }^{5}$ Address: ACT, Inc., 500 ACT Drive, lowa City, lowa, 52243, USA

\section{Background and Motivation}

The past 70 years of research in learning has primarily favoured a cognitive perspective. As such, learning and learning performance were measured based on factors such as memory, encoding, and retrieval. More sophisticated learning activities, such as perspective changes, still relied on a fundamental cognitive architecture (Dunlosky \& Rawson, 2019). Early researchers advocating for a constructivist learning lens, such as Piaget, also assessed development on a range of cognitive tasks. Over the past several decades, this view of learning as cognitive has given rise to a range of augmenting perspectives. Researchers increasingly focus on mindsets, social learning, peer effects, self-regulation, and self-perception to evaluate the broader scope of learning. For learning analytics (LA), this transition has important implications for data collection and analysis, tools and technologies used, research design, and experimentation. This special issue continues existing conversations around LA and emerging competencies (Dawson \& Siemens, 2014; Buckingham Shum \& Crick, 2016) but also reflects the growing number of researchers engaging with these topics.

When we first started planning this special issue, we grappled with the terms that best encapsulated the extension of learning sciences and LA research beyond only cognition. Some of the considered terms are noncognitive, twenty-first-century competencies, soft skills, employability skills, and social and emotional learning. After extensive review of the literature, including the articles in this special issue, it became clear that our difficulty in settling on a term was hardly unique. There is little consensus among researchers and practitioners on what to call this cluster of concepts (Matteson, Anderson, \& Boyden, 2016). As a result, we use the term social and emotional learning (SEL), but we do so awkwardly. It is an incomplete term to explain the scope of learning outside traditional cognitive lenses. Instead, we argue that a range of terms will be needed, with limited chance of a unifying term in the near future. This in itself is instructive of the growing number of researchers grappling with these concepts and how underexplored the space currently is. The lack of a common term, however, has not reduced interest across a range of sectors attempting to understand, quantify, assess, support, and promote these skills and attributes (Farrington et al., 2012; Rosen, Glennie, Dalton, Lennon, \& Bozick, 2010), as well as their impact on academic and career success (Berenson, Boyles, \& Weaver, 2008; Collet, du Plessis, \& Hine, 2015; Deming, 2017; Pulakos, Arad, Donovan, \& Plamondon, 2000), in a range of formal and informal settings.

Assessment of SEL remains problematic. As noted in our special issue call, these concepts "capture dimensions of learning broader than academic knowledge and have been well established in contemporary literature as highly relevant for success in school, work, or life in general. However, a challenge remains with the current assessment practices (e.g., summative, selfreported, infrequent, subjective) of these constructs as they are hard to quantify and measure." We arrive at something that should be motivating for LA researchers: a concept that has significant interest in a range of educational settings, remains a largely unexplored area of research, and promises to significantly influence how learning is assessed and promoted in classrooms, in the workplace, and online. The space needs thoughtful and deliberate exploration.

\section{History of SEL and Learning Analytics and Knowledge (LAK)}

Recognizing the need for future skills that promote collaboration, engagement, and lifelong learning, learning systems from $\mathrm{K}-12$ through to corporate learning are including social and emotional dimensions of learning in their curriculum. This is where the LA research community comes in, specifically in defining how to develop these dispositions that would lead to success in a complex changing world. Researchers in LA have made some initial attempts to define SEL and provide ways to assess competencies that are highly valued by educational institutions in a special issue of The Journal of Learning Analytics (Buckingham Shum \& Crick, 2016). This early focus on SEL in LA was further broadened through SEL workshops. The inaugural workshop was delivered in conjunction with the annual Learning Analytics and Knowledge (LAK) conference in 2018. The first workshop focused on SEL assessment at scale while aiming to bridge the scarcity of scalable SEL skills assessments in digital environments. The second workshop, at LAK'19, further enriched the goal by incorporating the

ISSN 1929-7750 (online). The Journal of Learning Analytics works under a Creative Commons License, Attribution — NonCommercial-NoDerivs 3.0 Unported (CC BY-NC-ND 3.0) 
promotion of inclusion and diversity. The third workshop aims to systematically examine how LA methods can be used to evaluate SEL and, conversely, how understanding student SEL skills can improve LA models and the impact of interventions driven by these models.

In line with LAK'20's theme of "shaping the future of the field," the third workshop aims at investigating how LA can enable more impactful SEL research by (1) offering tutorials to highlight existing work that incorporates various LA methods into measuring and predicting critical SEL attributes and (2) connecting researchers and practitioners to collaboratively advance and expand this field by envisioning future activities and development as a deliverable of this workshop.

Over the past three years, SEL in the field of LAK has grown from an emergent topic to a growing branch of interest. The workshop attendees have showcased their early research and proposals on how SEL links to student learning and how the SEL focus can shape the development of LAK. The sophistication of both the topics and the methodologies advances year over year. Because of increasing demand and broadening interest in the area, we must invite scholars in the LA community to collaborate on formal exploration of SEL research. As such, the workshop organizers decided to host this Journal of Learning Analytics special issue to raise the profile of SEL research and invite increased consideration by the LA community of identification, assessment, and support for social and emotional dimensions of learning.

In the first article, Porter et al. look at how learners persist in the face of challenges. Confusion and difficulty are often part of the learning process and can contribute positively (Baker, D'Mello, Rodrigo, Ma, \& Graesser, 2010; D'Mello \& Graesser, 2012). Difficulties, however, can also lead to discouragement and disengagement, as discussed in Porter and her colleagues' paper. The researchers use an adapted persistence, effort, resilience, and challenge-seeking (PERC) task to assess learner performance in online settings. They describe the limitations of traditional questionnaires and focus instead on mastery behaviours because they are more accurate at capturing learning performance. As the authors state, PERC "can contribute to the comprehensive and multimethod study of mastery behaviours to shed greater light on how they develop, and how to foster them." Additionally, Porter et al. state that their "PERC is the only performance task of aggregate mastery behaviours to demonstrate evidence of convergent, discriminant, and incremental predictive validity." Why does this matter? Understanding the behaviours of adolescent learners (the population of their study) in the face of difficulty is a needed foundation for assessing the impact of specific behaviours, the types of interventions that are most effective, and the ways that scaffolding can be introduced at critical times.

In the second article, Crossley, Karumbaiah, Ocumpaugh, Labrum, \& Baker evaluate SEL in the domain-specific context of mathematics. They emphasize existing research in mathematics success that indicates that a range of patterns, such as sophistication and sentiment, can be assessed based on language production. But can language be used to get at more ambiguous constructs such as affect? Crossley et al. suggest that it is possible when bringing together multiple sources of data and analytics strategies, including survey, clickstream, and natural language processing (NLP). Their focus is on math identity, "the degree to which one considers oneself a "math person," and how this can be "decomposed" into a series of finer-grained constructs that enable them to target those attributes through surveys and then ultimately identify and assess noncognitive factors involved in math success. While their study focuses on educational software for learning math, their multimodal approach (clickstream, language, and survey data) for examining noncognitive variables will be of interest to researchers in a range of disciplines.

In the third article, Dowell, Lin, Godfrey, \& Brooks explore collaborative problem solving (CPS), a setting in which SEL is particularly prominent. The pedagogical approaches of many university classrooms are shifting toward active learning because engaged practices produce better educational outcomes than traditional lecture formats (Freeman et al., 2014). As learning on campuses becomes more social, collaborative, and group based, new approaches to assess the factors that contribute to performance are required. Using group communication analysis (GCA) (Dowell, Nixon, \& Graesser, 2019), the authors use a range of sociocognitive measures to create interaction profiles evident in CPS. They highlight that existing research in complex problem-solving addresses the behaviours and actions of members but has not yet detailed the sociocognitive attributes that contribute to success in CPS. Their evaluation reflects an important advancement in modelling both how researchers might break down complex social constructs into a research program and how SEL is reflected in group learning. It is vital that tools and methods such as GCA be developed to move SEL from an ambiguous construct to a research-informed model.

Finally, Kent \& Cukurova shift attention from the individual learner to the collective group and focus specifically on the processes of collaboration. At all levels of the education system, assessment focuses on individual gains. Where group work or collaborative learning is utilized, the assessment of individuals is still a primary goal. SEL requires interaction and engagement with others. This means researchers will need better tools for assessing collaboration, knowledge gains in groups, and successful configurations of networks. One approach to begin assessing collective learning benefits from working together is to use collaborative cognitive load theory (CCLT), as Kent \& Cukurova have done in their paper. Cognitive load is not seen solely as an individual construct but rather as one that has a corollary role in a collective. The authors utilize social network

ISSN 1929-7750 (online). The Journal of Learning Analytics works under a Creative Commons License, Attribution — NonCommercial-NoDerivs 3.0 Unported (CC BY-NC-ND 3.0) 
analysis and introduce the $\mathrm{CLaP}$ (collaborative learning as a process) index to better understand the processes of collaborative learning. When we treat group processes as more than just the outcome of individual interactions, that is, a separate entity that has coordination costs, working memory, and related attributes, the direct impact on SEL quickly becomes evident. We are more than individual learners. The networks that we form contain knowledge and have a memory structure that influences how quickly a group achieves success and how well they perform.

\section{Future Development: Where Might Things Go?}

The four articles in the special issue advance research on SEL by providing models and frameworks that can be used to identify and assess attributes in social emotional learning. Integrated methods (Crossley et al.); validated models of learner behaviour in challenging settings (Porter et al.); the influence of individual profiles on complex problem-solving (Dowell et al.); and the focus on the collective group as possessing cognitive structures, including limitations (Kent \& Cukurova), provide the LA community with promising approaches to identifying, assessing, and engaging with SEL.

Questions arise, however, about longer-term implications. Identifying and assessing SEL is a needed foundation. Future focus will be on how to develop and improve both individual and group skills. This will require attention to a range of areas, including the following.

Data Sources. Existing SEL studies often look at survey measures of SEL constructs and how these relate to student grades. Behavioural data, such as those derived from learning management systems (LMSs), are already being incorporated into the investigation, as noted by the papers in this issue. More advanced data collection, such as psychophysiological, will be needed to understand SEL, including temporal changes and variations distinct to individuals (such as during different times of the day or in different topic areas).

Outcomes. Academic grades are the most popular outcome measures in SEL research studies. There is a growing need to investigate how these SEL constructs contribute to success beyond academic settings, such as workplaces and well-being. Given the heavy "evidence-centred" mindset of education and corporations, how tolerant will they be to attributes that have a longer timeline and less-quantifiable outcomes? Shaping this conversation to ensure that the value of SEL is recognized beyond a grade metric will be an important contribution from LA researchers.

Generalizability. The majority of the SEL studies are grounded in the $\mathrm{K}-12$ context. We will need to carefully investigate whether the findings apply to other populations, such as adult learners and online learners. In this special issue, all four papers have an online or a digital component, and two focus on adult learning settings (Kent \& Cukurova; Dowell et al.). Applying the tools and methods presented to other contexts is a logical next step.

Interventions. As researchers clarify the conditions under which SEL flourishes and the distinct attributes that contribute to successful learning, practical applications arise regarding the role of interventions and the developmental nature of SEL. When students aren't performing well, what interventions are required? Existing literature has identified a range of approaches to assist learners when they cognitively underperform. What is the equivalent in SEL? As well, to what degree can we develop learner SEL skills and mindsets through our existing pedagogical approaches? What needs to change in classrooms to make that a more explicit focus?

In addition to advancing basic science in learning, LA directly connects to practice and implementation. Few topics are more prevalent in today's education settings than future skills and mindsets, including their assessment and development. Systems of learning, whether K-12, higher education, or lifelong learning, represent a network of networks. A change in one aspect of the system generates consequences in others. The growing focus on SEL reflects these systemic ripples: new pedagogical approaches are being adopted, active learning is promoted, and collaborative and team-based learning forms an important aspect of any student learning experience. We invite LA researchers to engage with these promising areas of research and practice through the lens of SEL.

\section{References}

Baker, R. S. J. d., D’Mello, S. K., Rodrigo, Ma. M. T., \& Graesser, A. C. (2010). Better to be frustrated than bored: The incidence, persistence, and impact of learners' cognitive-affective states during interactions with three different computer-based learning environments. International Journal of Human-Computer Studies, 68(4), 223-241. https://dx.doi.org/10.1016/j.ijhcs.2009.12.003

Berenson, R., Boyles, G., \& Weaver, A. (2008). Emotional intelligence as a predictor of success in online learning. The International Review of Research in Open and Distributed Learning, 9(2). https://doi.org/10.19173/irrodl.v9i2.385

Buckingham Shum, S., \& Crick, R. D. (2016). Learning analytics for 21st century competencies. Journal of Learning Analytics, 3(2), 6-21. https://dx.doi.org/10.18608/jla.2016.32.2

Collet, C., du Plessis, K., \& Hine, D. (2015). Employability skills: Perspectives from a knowledge-intensive industry. 
Education + Training, 57(5), 532-559. https://dx.doi.org/10.1108/ET-07-2014-0076

Dawson, S., \& Siemens, G. (2014). Analytics to literacies: The development of a learning analytics framework for multiliteracies assessment. The International Review of Research in Open and Distributed Learning, 15(4). https://dx.doi.org/10.19173/irrodl.v15i4.1878

Deming, D. J. (2017). The growing importance of social skills in the labor market. The Quarterly Journal of Economics, 132(4), 1593-1640. https://dx.doi.org/10.1093/qje/qjx022

D’Mello, S., \& Graesser, A. (2012). Dynamics of affective states during complex learning. Learning and Instruction, 22(2), 145-157. https://dx.doi.org/10.1016/j.learninstruc.2011.10.001

Dowell, N. M. M., Nixon, T. M., \& Graesser, A. C. (2019). Group communication analysis: A computational linguistics approach for detecting sociocognitive roles in multiparty interactions. Behavior Research Methods, 51(3), 1007-1041. https://dx.doi.org/10.3758/s13428-018-1102-z

Dunlosky, J., \& Rawson, K. A. (Eds.). (2019). The Cambridge Handbook of Cognition and Education. Cambridge University Press. https://dx.doi.org/10.1017/9781108235631

Farrington, C. A., Roderick, M., Allensworth, E., Nagaoka, J., Keyes, T. S., Johnson, D. W., \& Beechum, N. O. (2012). Teaching adolescents to become learners: the role of noncognitive factors in shaping school performance - A critical literature review. Consortium on Chicago School Research. https://consortium.uchicago.edu/publications/teachingadolescents-become-learners-role-noncognitive-factors-shaping-school

Freeman, S., Eddy, S. L., McDonough, M., Smith, M. K., Okoroafor, N., Jordt, H., \& Wenderoth, M. P. (2014). Active learning increases student performance in science, engineering, and mathematics. Proceedings of the National Academy of Sciences, 111(23), 8410-8415. https://dx.doi.org/10.1073/pnas.1319030111

Matteson, M. L., Anderson, L., \& Boyden, C. (2016). "Soft skills": A phrase in search of meaning. Portal: Libraries and the Academy, 16(1), 71-88. https://dx.doi.org/10.1353/pla.2016.0009

Pulakos, E. D., Arad, S., Donovan, M. A., \& Plamondon, K. E. (2000). Adaptability in the workplace: Development of a taxonomy of adaptive performance. Journal of Applied Psychology, 85(4), 612-624. https://dx.doi.org/10.1037/0021$\underline{9010.85 .4 .612}$

Rosen, J. A., Glennie, E. J., Dalton, B. W., Lennon, J. M., \& Bozick, R. N. (2010). Noncognitive skills in the classroom: New perspectives on educational research. RTI International. https://www.rti.org/sites/default/files/resources/bk-00041009-rosen.pdf 